\title{
How much tumor volume is responsible for development of clinical symptoms in patients with convexity, parasagittal, and falx meningiomas?
}

\section{Shuhei Yamada}

Osaka University: Osaka Daigaku

Noriyuki Kijima ( $\square$ norikijima@gmail.com )

Osaka University Graduate School of Medicine https://orcid.org/0000-0003-2103-1909

\section{Tomoyoshi Nakagawa}

Osaka University: Osaka Daigaku

Ryuichi Hirayama

Osaka University: Osaka Daigaku

\section{Manabu Kinoshita}

Osaka University: Osaka Daigaku

\section{Naoki Kagawa}

Osaka University: Osaka Daigaku

Haruhiko Kishima

Osaka University: Osaka Daigaku

\section{Research Article}

Keywords: convexity meningioma, falx meningioma, parasagittal meningioma, symptomatic progression, tumor volume

Posted Date: April 21st, 2021

DOI: https://doi.org/10.21203/rs.3.rs-437289/v1

License: (c) (i) This work is licensed under a Creative Commons Attribution 4.0 International License. Read Full License

Version of Record: A version of this preprint was published at Frontiers in Neurology on November 17th, 2021. See the published version at https://doi.org/10.3389/fneur.2021.769656. 


\section{Abstract \\ Purpose}

Meningiomas are the most common primary intracranial neoplasms. Their volume and location are important factors related to the appearance of clinical symptoms. This study aimed to identify factors that influence clinical symptoms and to determine a specific threshold tumor volume for the prediction of symptomatic progression in patients with convexity, parasagittal, and falx meningiomas.

\section{Methods}

We retrospectively studied patients with radiologically suspected convexity, parasagittal, or falx meningiomas at our institution.

\section{Results}

The data of three hundred thirty-three patients were analyzed. We further divided patients into two groups based on clinical symptoms: as asymptomatic group (250 cases) and a symptomatic group (83 cases). Univariate analysis revealed significant differences between the groups in terms of sex $(p=0.002)$, age at the time of volumetric analysis $(p<0.001)$, hyperintense lesions on T2-weighted images $(p=0.029)$, peritumoral edema $(p<0.001)$, maximum tumor diameter $(p<0.001)$, and tumor volume $(p<0.001)$. Further multivariate analysis revealed significant differences between the groups in terms of age at the time of volumetric analysis $(p=0.002)$, peritumoral edema $(p<0.001)$, and tumor volume $(p<0.001)$. Receiver operating characteristic curve revealed a threshold tumor volume of $21.1 \mathrm{ml}$ for predicting whether a patient would develop symptoms (sensitivity 0.843 , specificity 0.880 , an area under the curve 0.919 [95\% confidence interval: 0.887-0.951]).

\section{Conclusion}

We identified factors predictive of clinical symptoms in patients with convexity, parasagittal, and falx meningiomas and determined the first-ever threshold tumor volume for predicting symptomatic progression in such patients.

\section{Introduction}

Meningiomas are the most common primary intracranial tumors, accounting for approximately $25 \%-38 \%$ of all such lesions $[1,2]$. The number of incidentally discovered meningiomas has increased with the widespread use of neuroimaging modalities such as computed tomography and magnetic resonance imaging (MRI). In fact, radiological studies have revealed that neuroimaging could incidentally reveal suspected meningioma lesions with an incidence ranging from $0.9 \%$ to $2.5 \%$ in individuals aged in their 
middle years and older [3,4]. On the other hand, meningiomas are often discovered because of a variety of symptoms, including motor and sensory deficits, cognitive decline, and epilepsy [5-7]. However, the factors that determine whether a lesion is symptomatic remain unclear.

Meningiomas are benign neoplasms that can exhibit a variety of growth patterns [8,9] and, eventually, $67 \%-75 \%$ of them enlarge $[6,9,10]$. In one study, tumor volume was statistically significantly related to the appearance of clinical symptoms [5]. In recent meta-analyses, the authors observed that $4.7 \%-8.1 \%$ of patients with incidentally discovered intracranial meningiomas developed related symptoms at follow-up visits $[10,11]$. However, the specific locations of the tumors were not examined in either report. The location of such a tumor is important as it is related to the symptoms a patient will experience [7], as well as the clinical and biological behavior of the tumor $[12,13]$.

Convexity, parasagittal, and falx meningiomas account for almost $50 \%$ of all meningiomas [14]. In the report in which the association between tumor volume and clinical symptoms were observed in intracranial meningiomas, nearly half of the cases were skull-base meningiomas [5]. Convexity, parasagittal, and falx meningiomas differ from skull-base meningiomas in that they are located in the supratentorial space and do not involve cranial nerves. Thus, the tumor volume that causes clinical symptoms differs between supratentorial and skull-base meningiomas, and it is important to analyze the tumor volume that causes clinical symptoms exclusively for convexity, parasagittal, and falx meningiomas.

The purpose of this retrospective study was to identify factors that influence clinical symptoms and to determine a specific threshold tumor volume for the prediction of symptomatic progression in patients with convexity, parasagittal, and falx meningiomas.

\section{Methods}

\section{Study design and patient selection}

We conducted a retrospective case-control study of patients with radiologically suspected convexity, parasagittal, or falx meningiomas. We collected data from patients whose first visit was from 1990 to 2020 at our institution. We excluded patients diagnosed with neurofibromatosis, those for whom MRI Digital Imaging and Communications in Medicine (DICOM) data were not available, and those whose symptoms were unknown due to insufficient medical records of their first visit. When patients had more than one convexity, parasagittal, or falx meningioma, we selected the largest one for analysis. The Osaka University Clinical Research Review Committee approved the study (approval number 14231) and waived the need for written informed consent, as all data were retrospective.

\section{Definition of symptoms}

We determined from their medical records whether patients had clinical findings, which we defined as clinical symptoms. We further defined neurological symptoms as excluding epilepsy or non-specific 
symptoms such as headache.

\section{Volumetric analysis}

We measured the volume of each lesion using the latest MRI DICOM data for asymptomatic patients or the MRI DICOM data at the time of symptom onset for symptomatic patients. We used Horos for macOS to perform the measurements (Horos is a free and open-source code software program that is distributed free of charge under the LGPL license at Horosproject.org and sponsored by Nimble Co LLC $\mathrm{d} / \mathrm{b} / \mathrm{a}$ Purview in Annapolis, MD USA). We measured the tumor area in each slice by manually tracing the tumor boundary. Thereafter, we multiplied the sum of all the areas by the thickness between slices, including the gaps.

\section{Tumor diameter}

We used the same MRI DICOM data as for volumetric analysis to measure tumor diameter. The maximum tumor diameter was determined using either axial, coronal, or sagittal images.

\section{Tumor location and side}

The lead author (S.Y.) carefully determined the locations of the lesions via MRI, which was independently confirmed by the senior author (N.K.). We divided tumor location in two ways: convexity, parasagittal angle, and falx cerebri; or right, midline, and left.

\section{Interpretation of T2-weighted images}

We classified lesions as hyperintense if the mean signal intensity of the tumor was at least 1.3 times higher than that of the deep white matter on T2-weighted images (T2WIs). Other lesions were classified as "others," including those that were too heterogeneous to classify. One case was excluded from analysis because the lesion was too small to evaluate.

\section{Statistical analysis}

All statistical analyses were performed using R 3.6.3 for Windows (www.R-project.org; R Foundation for Statistical Computing, Vienna, Austria). Statistical differences for categorical variables were examined using a two-tailed Fisher's exact test. Continuous variables were assessed using the Mann-Whitney $\mathrm{U}$ test. Multivariate logistic regression analysis was performed with variables that were significant in those univariate analyses. The thresholds were calculated by receiver operating characteristic (ROC) analysis using the Youden index. Probability values $<0.05$ were considered significant.

\section{Results}

Overall patient cohort 
Figure 1 illustrates the flow of patient selection. The data of three hundred thirty-three patients (84 male and 249 female) were analyzed. The median age at volumetric analysis was 70 years (range 23-90 years). We further divided patients into two groups based on clinical symptoms: an asymptomatic group (250 cases) and a symptomatic group (83 cases).

Comparison between the asymptomatic and symptomatic groups

Table 1 summarizes the characteristics of each group. Univariate analysis revealed significant differences between the groups in terms of sex $(p=0.002)$, age at the time of volumetric analysis $(p<$ $0.001)$, hyperintense lesions on T2Wls $(p=0.029)$, peritumoral edema $(p<0.001)$, maximum tumor diameter $(p<0.001)$, and tumor volume $(p<0.001$, Figure $2 a)$. For multivariate analysis, the maximum tumor diameter was excluded as it is a similar metric to tumor volume [15]. Multivariate analysis revealed significant differences between the groups in terms of age at the time of volumetric analysis $(p=0.002)$, peritumoral edema $(p<0.001)$, and tumor volume $(p<0.001)$. The odds ratio for peritumoral edema was 5.94 (95\% confidence interval [Cl]: 2.74-12.86).

\section{Thresholds for predicting development of symptoms}

Patients in the symptomatic group experienced motor deficits (37 cases), epilepsy (18 cases), gait disorder ( 7 cases), visual impairment (7 cases), cognitive decline (6 cases), aphasia (4 cases), headaches ( 2 cases), sensory deficits ( 1 case), and a subcutaneous mass ( 1 case). The ROC curve revealed a threshold tumor volume of $21.1 \mathrm{ml}$ for predicting whether a patient would develop symptoms, with a sensitivity of 0.843 , a specificity of 0.880 , and an area under the curve (AUC) of 0.919 (Figure $2 \mathrm{~b}, 95 \% \mathrm{Cl}$ : 0.887-0.951). In addition, a threshold maximum tumor diameter of $40 \mathrm{~mm}$ may also be a reliable marker for predicting which patients will become symptomatic, with a sensitivity of 0.819 , a specificity of 0.840 , and an AUC of 0.893 (95\% Cl: 0.856-0.930). For 13 patients in the symptomatic group, we obtained MRI DICOM data when they had been asymptomatic at their first visit. Of these, the $21.1-\mathrm{ml}$ and $40-\mathrm{mm}$ threshold correctly predicted the development of symptoms in seven cases (54\%).

\section{Threshold for predicting development of neurological symptoms}

When focusing only on neurological symptoms (62 cases), the threshold for tumor volume was also 21.1 $\mathrm{ml}$ (sensitivity 0.871 , specificity 0.880 , AUC 0.937 [95\% Cl: $0.910-0.965]$ ). The threshold for maximum tumor diameter for prediction of neurological symptoms was almost the same as that for all symptoms: $41 \mathrm{~mm}$ (sensitivity 0.839 , specificity 0.856 , AUC 0.914 [95\% Cl: 0.880-0.948]).

\section{Correlation between tumor volume and tumor side}

Table 2 displays the difference in tumor volume between the asymptomatic and symptomatic groups, depending on the side where the tumor was located. On each side, the tumor volume was significantly larger in the symptomatic than in the asymptomatic group $(p<0.001)$. The threshold for predicting patients in which symptoms would develop was similar: $21.1 \mathrm{ml}$ for right-sided and $22.7 \mathrm{ml}$ for left-sided meningiomas. 
Thirty-three patients in the asymptomatic and 77 in the symptomatic group received surgical treatment. Of the 110 patients, the meningiomas of $90(81.8 \%)$ were World Health Organization (WHO) grade I, 10 $(9.1 \%)$ were WHO grade II, 3 (2.7\%) were WHO grade III, and 7 (6.4\%) were not mentioned in WHO grade. There were no significant differences between patients with WHO grade I and those with WHO grade II/III meningiomas, except in the maximum tumor diameter, which was larger in the latter than in the former (Table 3).

\section{Discussion}

In the present study, we identified factors that are related to clinical symptoms of patients with convexity, parasagittal, and falx meningiomas, and, to our knowledge, we determined the first-ever threshold of tumor volume for predicting symptomatic progression in such patients. This may allow clinicians to predict when a growing, asymptomatic meningioma will become symptomatic. Currently, observation is the first choice for management of patients with asymptomatic meningiomas [16]; these results may be useful to determine the necessity of treatment and its appropriate timing.

In this study, we also determined a threshold maximum tumor diameter of $40 \mathrm{~mm}$ for the prediction of symptomatic progression of patients (sensitivity 0.819 , specificity 0.840 , AUC 0.893 ). However, a tumor volume of $21.1 \mathrm{ml}$ was a more accurate threshold (sensitivity 0.843 , specificity 0.880 , AUC 0.919 ). Maximum tumor diameter and tumor volume are highly correlated [15]. However, tumors do not always grow in the direction of their maximum diameter, which may be why we discovered tumor volume to be the more accurate predictive factor. Maximum tumor diameter is one of the most convenient clinical metrics; however, our results indicate that the development and widespread use of a simple method to measure tumor volume is needed.

Meningiomas manifest in a variety of symptoms [7], including non-specific symptoms such as headaches and dizziness [6]. In this study, we also calculated thresholds for tumor volume and maximum tumor diameter to predict the development of neurological symptoms. However, the thresholds were similar to those for all clinical symptoms. Therefore, it may be less important for clinicians to examine whether non-specific symptoms are caused by a given meningioma.

The hemisphere in which glioblastomas and strokes occur affects the symptoms that the patient experiences $[17,18]$; however, in this study of meningiomas, we detected no differences in the threshold of tumor volume regardless of the tumor localization. This may be because few patients presented with cognitive decline $(7 \%)$ or aphasia (5\%), both of which may depend on the affected hemisphere. Therefore, further studies are needed to validate our findings.

We should note that this study has several limitations. The first is the fact that this was a single-center, retrospective study conducted in Japan. As Japan has the largest number of MRIs per unit population in the world [19], a larger proportion of small, asymptomatic meningiomas may be detected than in other 
countries. This would have lowered the thresholds of tumor volume and maximum tumor diameter for predicting symptomatic progression of patients in this study. The second limitation is the possibility of errors in volumetric measurements. Volumetric measurement may be inaccurate especially for small tumors [20], and manual segmentation may be inconsistent [21]. Finally, this study was conducted on radiologically presumed meningiomas; therefore, $2.9 \%-3.4 \%$ of our study population may actually have had other tumors $[9,22]$.

\section{Conclusion}

In the present study, we identified factors predictive of clinical symptoms in patients with convexity, parasagittal, and falx meningiomas and, to our knowledge, determined the first-ever threshold tumor volume for predicting symptomatic progression in such patients. These results may be useful in allowing clinicians to estimate when a growing, asymptomatic meningioma will develop clinical symptoms, thereby improving management of patients with the disease.

\section{Declarations}

\section{Funding}

Not applicable

\section{Conflicts of Interest}

We declare that we have no conflict of interest.

Availability of data and material

Data are available upon reasonable request.

Code availability

Not applicable

\section{Authors' contributions}

SY and NoK contributed to the concept, drafting, and writing the manuscript. TN contributed to the acquisition of date. $\mathrm{RH}$ and NaK contributed to the manuscript editing. MK contributed to the provision of insightful thought and design. HK contributed to the study supervision.

\section{Ethics approval}

The Osaka University Clinical Research Review Committee approved the study (approval number 14231). 
The Osaka University Clinical Research Review Committee waived the need for written informed consent.

Consent for publication

Not applicable

\section{Acknowledgments}

We would like to thank Editage (www.editage.com) for English language editing.

\section{References}

1. Claus EB, Bondy ML, Schildkraut JM, et al (2005) Epidemiology of intracranial meningioma. Neurosurgery 57:1088-1094. https://doi.org/10.1227/01.NEU.0000188281.91351.B9

2. Ostrom QT, Patil N, Cioffi G, et al (2020) CBTRUS Statistical Report: Primary Brain and Other Central Nervous System Tumors Diagnosed in the United States in 2013-2017. Neuro Oncol 22:iv1-iv96. https://doi.org/10.1093/neuonc/noaa200

3. Vernooij MW, Ikram MA, Tanghe HL, et al (2007) Incidental Findings on Brain MRI in the General Population. N Engl J Med 357:1821-1828. https://doi.org/10.1056/nejmoa070972

4. Bos D, Adams HHH, Zonneveld HI, et al (2016) Course of Incidental Findings on Brain MR Images: The Population-. Radiology 000:1-9

5. Kauke M, Safi AF, Stavrinou P, et al (2019) Does Meningioma Volume Correlate With Clinical Disease Manifestation Irrespective of Histopathologic Tumor Grade? J Craniofac Surg 30:e799-e802. https://doi.org/10.1097/SCS.0000000000005845

6. Kim KH, Kang SJ, Choi J-W, et al (2019) Clinical and radiological outcomes of proactive Gamma Knife surgery for asymptomatic meningiomas compared with the natural course without intervention. J Neurosurg 130:1740-1749. https://doi.org/10.3171/2017.12.JNS171943

7. Fathi AR, Roelcke U (2013) Meningioma. Curr Neurol Neurosci Rep 13:. https://doi.org/10.1007/s11910-013-0337-4

8. Hashiba T, Moto NH, Izumoto S, et al (2009) Serial volumetric assessment of the natural history and growth pattern of incidentally discovered meningiomas: Clinical article. J Neurosurg 110:675-684. https://doi.org/10.3171/2008.8.JNS08481

9. Behbahani M, Skeie GO, Eide GE, et al (2019) A prospective study of the natural history of incidental meningioma-Hold your horses! Neuro-Oncology Pract 6:438-450. https://doi.org/10.1093/nop/npz011

10. Nakasu S, Nakasu Y (2020) Natural history of meningiomas: Review with meta-analyses. Neurol Med Chir (Tokyo) 60:109-120. https://doi.org/10.2176/nmc.ra.2019-0213

11. Islim Al, Mohan M, Moon RDC, et al (2019) Incidental intracranial meningiomas: a systematic review and meta-analysis of prognostic factors and outcomes. J Neurooncol 142:211-221. https://doi.org/10.1007/s11060-019-03104-3 
12. Maiuri F, Mariniello G, Guadagno E, et al (2019) WHO grade , proliferation index, and progesterone receptor expression are different according to the location of meningioma. 2553-2561

13. Hashimoto N, Rabo CS, Okita Y, et al (2012) Slower growth of skull base meningiomas compared with non-skull base meningiomas based on volumetric and biological studies. J Neurosurg 116:574-580. https://doi.org/10.3171/2011.11.JNS11999

14. Sun C, Dou Z, Wu J, et al (2020) The Preferred Locations of Meningioma According to Different Biological Characteristics Based on Voxel-Wise Analysis. Front Oncol 10:1-12. https://doi.org/10.3389/fonc.2020.01412

15. Ishi Y, Terasaka S, Yamaguchi S, et al (2016) Reliability of the Size Evaluation Method for Meningiomas: Maximum Diameter, ABC/2 Formula, and Planimetry Method. World Neurosurg 94:80-88. https://doi.org/10.1016/j.wneu.2016.06.108

16. Goldbrunner R, Minniti G, Preusser M, et al (2016) EANO guidelines for the diagnosis and treatment of meningiomas. Lancet Oncol 17:e383-e391. https://doi.org/10.1016/S1470-2045(16)30321-7

17. Baumann C, Tichy J, Schaefer JH, et al (2020) Delay in diagnosing patients with right-sided glioblastoma induced by hemispheric-specific clinical presentation. J Neurooncol 146:63-69. https://doi.org/10.1007/s11060-019-03335-4

18. Foerch C, Misselwitz B, Sitzer M, et al (2005) Difference in recognition of right and left hemispheric stroke. Lancet 366:392-393. https://doi.org/10.1016/S0140-6736(05)67024-9

19. Matsumoto M, Koike S, Kashima S, Awai K (2015) Geographic distribution of CT, MRI and PET devices in Japan: A longitudinal analysis based on national census data. PLoS One 10:1-12. https://doi.org/10.1371/journal.pone.0126036

20. Pan HC, Cheng FC, Sun MH, et al (2007) Prediction of volumetric data errors in patients treated with Gamma Knife radiosurgery. Stereotact Funct Neurosurg 85:184-191. https://doi.org/10.1159/000101297

21. Mazzara GP, Velthuizen RP, Pearlman JL, et al (2004) Brain tumor target volume determination for radiation treatment planning through automated MRI segmentation. Int J Radiat Oncol Biol Phys 59:300-312. https://doi.org/10.1016/j.jirobp.2004.01.026

22. Jadid KD, Feychting M, Höijer J, et al (2015) Long-term follow-up of incidentally discovered meningiomas. Acta Neurochir (Wien) 157:225-230. https://doi.org/10.1007/s00701-014-2306-3

\section{Tables}

Table 1. Characteristics of 333 patients and tumors 


\begin{tabular}{|c|c|c|c|c|c|}
\hline \multirow[t]{2}{*}{ Variable } & \multirow{2}{*}{$\begin{array}{c}\text { Asymptomatic } \\
\text { group } \\
(\mathrm{n}=250)\end{array}$} & \multirow{2}{*}{$\begin{array}{c}\text { Symptomatic } \\
\text { group } \\
(\mathrm{n}=83)\end{array}$} & \multicolumn{2}{|c|}{$p$ value } & \multirow{2}{*}{$\begin{array}{l}\mathrm{OR} \\
(95 \% \\
\mathrm{CI})\end{array}$} \\
\hline & & & univariate & multivariate & \\
\hline Sex (Male / Female) & $52 / 198$ & $32 / 51$ & 0.002 & 0.140 & $\begin{array}{l}1.79 \\
(0.83- \\
3.86)\end{array}$ \\
\hline Age at volumetric analysis (yrs) $* 1$ & $72(23-90)$ & $63(31-89)$ & $<0.001$ & 0.002 & 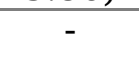 \\
\hline $\begin{array}{c}\text { Tumor location (Convexity / } \\
\text { Parasagittal / Falx) }\end{array}$ & $138 / 58 / 54$ & $35 / 25 / 23$ & 0.115 & - & - \\
\hline Tumor side (Right / Midline/ Left) & $122 / 6 / 122$ & $41 / 1 / 41$ & 1 & - & - \\
\hline MRI T2WI (Hyper / Others) *2 & $162 / 87$ & $65 / 18$ & 0.029 & 0.154 & $\begin{array}{c}1.82 \\
(0.80- \\
4.16)\end{array}$ \\
\hline Multiple lesions (Yes / No) & $22 / 228$ & $4 / 79$ & 0.345 & - & - \\
\hline Peritumoral edema (Yes / No) & $60 / 190$ & $69 / 14$ & $<0.001$ & $<0.001$ & $\begin{array}{r}5.94 \\
(2.74- \\
12.86)\end{array}$ \\
\hline$\underset{(\mathrm{mm}) * 1}{\operatorname{Maximum} \text { tumor diameter }}$ & $24(5-78)$ & $52(18-100)$ & $<0.001$ & - & - \\
\hline Tumor volume $(\mathrm{ml}) * 1$ & $5.0(0.1-148.0)$ & $\begin{array}{l}45.7(2.8- \\
188.9)\end{array}$ & $<0.001$ & $<0.001$ & - \\
\hline
\end{tabular}

*1 Median (range)

*2 Too small to evaluate: $\mathrm{n}=1$

95\% CI: 95\% confidence interval

Table 2. Correlation between tumor side and tumor volume (ml)

\begin{tabular}{rccccc}
\hline Tumor side & $\begin{array}{c}\text { Asymptomatic group } \\
(\mathrm{n}=244)\end{array}$ & $\begin{array}{c}\text { Symptomatic group } \\
(\mathrm{n}=82)\end{array}$ & $p$ value & AUC (95\% CI) & $\begin{array}{c}\text { Threshold } \\
(\mathrm{ml})\end{array}$ \\
\hline $\begin{array}{r}\text { Right } \\
(\mathrm{n}=163)\end{array}$ & $4.6(0.1-148.0)$ & $47.9(6.0-188.9)$ & $<0.001$ & $0.929(0.888-0.970)$ & 21.1 \\
\hline Left $(\mathrm{n}=163)$ & $5.5(0.2-129.7)$ & $44.7(2.8-158.1)$ & $<0.001$ & $0.903(0.851-0.955)$ & 22.7 \\
\hline
\end{tabular}

AUC: Area under the curve

95\% CI: 95\% confidence interval

\section{Figures}




\section{Convexity / Parasagittal / Falx meningiomas}

$$
n=404
$$

Excluded: $\mathrm{n}=71(17.6 \%)$

Neurofibromatosis:

$\mathrm{n}=5$

Lack of MRI DICOM data: $n=14$

Lack of symptoms data: $\quad n=52$

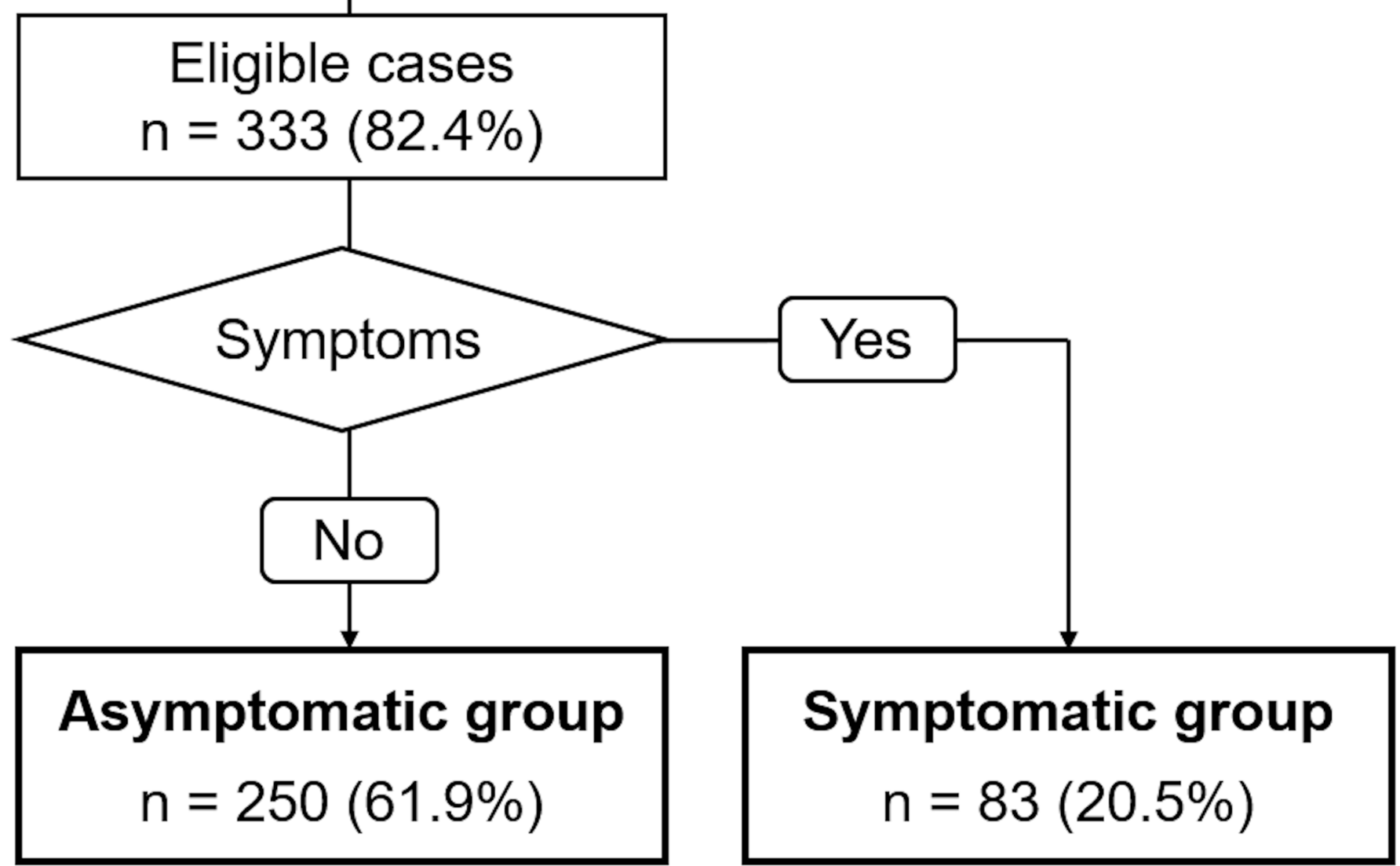

Figure 1

Flow of patient selection and classification 

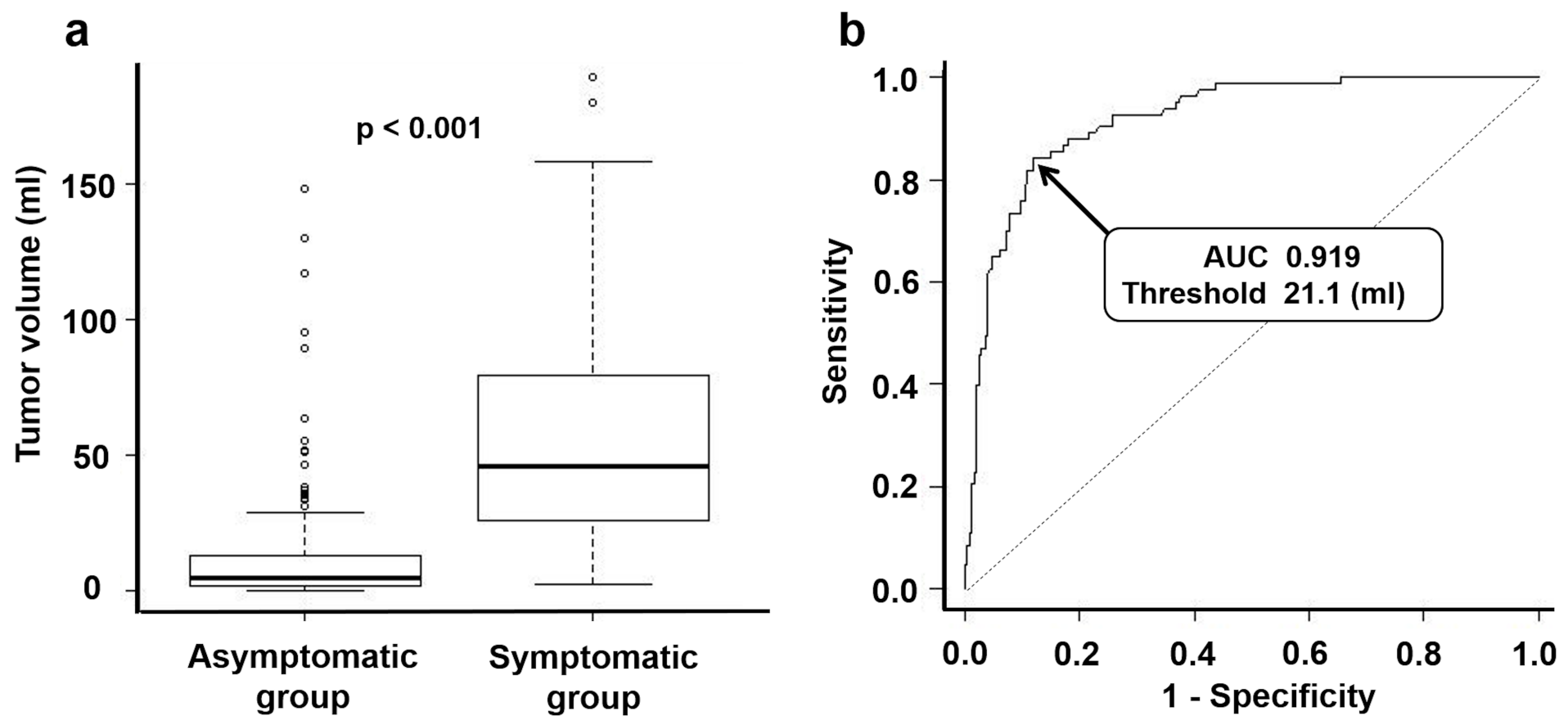

Figure 2

A) Box-and-whisker plots representing tumor volume of an asymptomatic group and symptomatic group The median tumor volume of an asymptomatic group and symptomatic group were $5.0 \mathrm{ml}$ and $45.7 \mathrm{ml}$, respectively. P-value for the Mann-Whitney U test: < 0.001 B) Receiver operating characteristic curve for predicting which patients will become symptomatic patient via tumor volume Area under the curve: 0.919 Threshold for tumor volume: $21.1 \mathrm{ml}$ 